matters arising

\section{Energy expenditure in children}

IT was with interest that we read the paper by Griffiths and Payne ${ }^{1}$, in which they presented interesting data and then discussed the possible relevance of environmental and genetic factors on the level and nature of the energy balance obtained with the two groups of children.

It is interesting to note that on expressing the mean expenditure and intake data for both groups as a ratio $\times 100$, precisely the same figure ensues

\section{$\frac{\text { Energy expenditure }}{\text { Energy intake }} \times 100$}

$$
\begin{array}{cc}
\text { Non-obese } & \text { Obese } \\
\frac{1,508}{1,433} \times 100= & \frac{1,174}{1,115} \times 100= \\
105.23 & 105.29
\end{array}
$$

Is it not possible therefore, that few overall metabolic differences exist between the 5-yr-old children of these two groups of parents, which by implication, suggests the predominance of environmental factors on these results?

It is surprising that the $\mathrm{O}$ group children were found to have lower daily energy intakes than their controls ( $N$ group). Therefore it would seem likely that parental pride during the nutritional survey may have had a marked influence.

We are also a little concerned that no comment was made about the data which showed that both groups of children were in negative energy balance, which for 5 yr olds would seem to be unlikely. From our experience SAMI measurements can be misleadingly high unless very precise and immobile electrical contacts are made, and thus background noise is eliminated.

\section{B. G. Miller W. R. OTro}

\section{Department of Physiology} and Biochemistry, University of Southampton, Southampton SO9 3TU, UK 1 Griffiths, M., and Payne, P. R., Nature, 260, 698-700
(1976).

Griffiths AND PAYNe REPLY-In our view the most important findings in our study were the differences in total daily energy metabolism, and of resting metabolic rate between the two groups of children.

We agree that the measurements of daily expenditure by the SAMI technique are inherently less reliable than are those of food energy intake, and have offered them as supporting evidence. That is to say, unlike Miller and Otto ${ }^{1}$, we regard the equal ratios of expenditure to intake as contributing positively to the evidence for different amounts of energy metabolised daily by the two groups.

Because the differences between the estimations of expenditure and intake (energy balance) are small in relation to the errors inherent in the two measurements, the energy balances do not statistically differ significantly from zero. We are not therefore inclined to attach any biological significance to the fact that they happen, on average, to be negative in both groups.

It is of course possible that one aspect of the behavioural differences between obese and normal people is that the former tend to 'cheat' when attempts are made to measure habitual intake, either their own or their children's. We can only repeat that: the $\mathrm{O}$ group children were not themselves measurably overweight so there was no obvious reason for the parents to take pride in suggesting that they eat sparingly, the degree of cooperation and the level of communication with the subjects about the objectives of the study were of necessity, very high, the admittedly less reliable estimates of expenditure closely parallel those of intakes, and show the same magnitude of difference between the groups.

\section{London School of Hygiene and Tropical Medicine} 1 Miller, B. G., and Otto, W. R., Nature, 263, 173
(1976).

\section{Benzodiazepines and GABA}

BASED on a few experiments on Deiters' neurones and cerebellar Purkinje cells, Steiner and Felix ${ }^{1}$ concluded that benzodiazepines antagonise the inhibitory effect of GABA. To understand the mode of action of centrally acting agents, the evidence available from various pharmacological experiments must be considered and the manifold pitfalls inherent in the single-cell recording and microelectrophoretic techniques taken into account. The conclusion of Steiner and Felix ${ }^{1}$ is in contrast to the well documented facts that benzodiazepines: (1) are the most potent antagonists of convulsions induced by picrotoxin and bicuculline $\mathrm{e}^{2-4}$ (generally accepted to block GABA receptors); (2) enhance presynaptic inhibition in the spinal cord and in the cuneate nucleus ${ }^{2,5,6} ;$ (3) enhance postsynaptic inhibition in the cuneate nucleus $^{6}$ and in the substantia nigra ${ }^{2,7}$ and (4) are mutual antagonists of GABA receptor blockers at inhibitory synaptic processes believed to be mediated by $\mathrm{GABA}^{5-7}$. We think that the conclusion of Steiner and Felix ${ }^{1}$ is based on the erroneous interpretation of experimental conditions that were inadequate for the specific problem.

In their experiments on vestibular neurones, they made the implicit assumption that the pulses applied to the cerebellar white substance (in their Fig. 1 either the time calibration is wrong or the rate of stimulation is not that indicated in the legend) released the same amount of GABA within the Deiters' nucleus before and after diazepam. This crucial premise is probably not correct for the following reasons. The stimulating electrode excites Purkinje cell axons directly and, in addition, evokes an unknown number of synaptically evoked discharges of Purkinje cells through the inevitable stimulation of cerebellar afferents. The number of the latter discharges depends on the balance between mono- and disynaptic excitation and the inhibitory influence on Purkinje cells. If diazepam, as we assume, enhances GABAmediated synaptic inhibition also in the cerebellar cortex, it should reduce the number of Purkinje cell discharges evoked synaptically by the stimulus train, and thus, produce an apparent reduction of the inhibitory effect on Deiters' neurones, which was interpreted by Steiner and Felix ${ }^{1}$ as an antagonism of the effect of GABA released within the vestibular nucleus. The apparent reduction by diazepam of the inhibitory effect of iontophoretically applied GABA may be explained in the same way. In the pre-drug period, the depression of Deiters' neurones is achieved by the fixed amount of 